Research report

\title{
Dawn simulation light impacts on different cognitive domains under sleep restriction
}

\author{
Virginie Gabel ${ }^{\mathrm{a}, *}$, Micheline Maire ${ }^{\mathrm{a}}$, Carolin F. Reichert ${ }^{\mathrm{a}}$, Sarah L. Chellappa ${ }^{\mathrm{a}, \mathrm{b}}$, \\ Christina Schmidt ${ }^{\mathrm{a}}$, Vanja Hommes ${ }^{\mathrm{c}}$, Christian Cajochen ${ }^{\mathrm{a}}$, Antoine U. Viola ${ }^{\mathrm{a}}$ \\ a Centre for Chronobiology, Psychiatric Hospital of the University of Basel, 4012 Basel, Switzerland \\ ${ }^{\mathrm{b}}$ Cyclotron Research Center, University of Liège, Liège, Belgium \\ c IT VitaLight IED PC Drachten, Philips Consumer Lifestyle, the Netherlands
}

\section{H I G H L I G H T S}

- We investigated morning light effects on cognition after a night of sleep restriction.

- Morning light effects depend on cognitive domain.

- Morning light effects depend on individual performance levels.

\section{A R T I C L E I N F O}

\section{Article history:}

Received 21 October 2014

Received in revised form

15 December 2014

Accepted 19 December 2014

Available online 27 December 2014

\section{Keywords:}

Dawn simulation light

Sleep restriction

Cognitive performance

\begin{abstract}
A B S T R A C T
Chronic sleep restriction (SR) has deleterious effects on cognitive performance that can be counteracted by light exposure. However, it is still unknown if naturalistic light settings (dawn simulating light) can enhance daytime cognitive performance in a sustainable matter.

Seventeen participants were enrolled in a 24-h balanced cross-over study, subsequent to SR (6-h of sleep). Two different light settings were administered each morning: a) dawn simulating light (DsL; polychromatic light gradually increasing from 0 to $250 \mathrm{~lx}$ during $30 \mathrm{~min}$ before wake-up time, with light around $250 \mathrm{~lx}$ for $20 \mathrm{~min}$ after wake-up time) and b) control dim light (DL; <8 lx). Cognitive tests were performed every $2 \mathrm{~h}$ during scheduled wakefulness and questionnaires were completed hourly to assess subjective mood.

The analyses yielded a main effect of "light condition" for the motor tracking task, sustained attention to response task and a working memory task (visual 1 and 3-back task), as well as for the Simple Reaction Time Task, such that participants showed better task performance throughout the day after morning DsL exposure compared to DL. Furthermore, low performers benefited more from the light effects compared to high performers. Conversely, no significant influences from the DsL were found for the Psychomotor Vigilance Task and a contrary effect was observed for the digit symbol substitution test. No light effects were observed for subjective perception of sleepiness, mental effort, concentration and motivation.

Our data indicate that short exposure to artificial morning light may significantly enhance cognitive performance in a domain-specific manner under conditions of mild SR.
\end{abstract}

(C) 2014 Elsevier B.V. All rights reserved.
Abbreviations: NIF, Non-Image Forming; fMRI, functional Magnetic Resonance Imaging; SR, sleep restriction; DsL, Dawn simulation light; DL, dim light; MTT, Motor Tracking Task; DSST, Digit Symbol Substitution Test; PVSAT, Paced Visual Serial Addition Task; simRT, Simple Reaction Time Task; SART, Sustained Attention to Response Task; PVT, Psychomotor Vigilance Task; V1-V2-V3, 1,2,3-back: Visual N-back Task; SC, superior colliculus.

* Corresponding author at: Psychiatric Hospital of University of Basel, Centre for Chronobiology, Wilhelm Klein-Strasse 27, CH-4018 Basel, Switzerland.

Tel.: +416132559 08; fax: +33676211028.

E-mail address: Virginie.gabel@upkbs.ch (V. Gabel).

\section{Introduction}

Numerous factors can influence cognitive performance, chief among them are the impact of time of day [1,2] and homeostatic sleep pressure [3]. Chronic sleep restriction (SR) has deleterious effects not only on daytime alertness but also on cognitive performance $[4,5]$.

Indeed, sleep disruption results in specific cognitive impairments including deficits in attention, executive function, non-declarative and declarative memory, as well as emotional reactivity and sensory perception [6-8]. Some studies show that 
light exposure can act as countermeasure for these cognitive impairments in humans $[9,10]$.

These acute impacts of light are usually referred to as nonvisual (or Non-Image Forming - NIF) effects, since they drift apart from classical involvement of rod and cone photopigments in visual responses to light. NIF light effects at shorter wavelength via novel photoreceptors containing the photopigment melanopsin appear to strongly impact the human circadian timing system $[11,12]$. Behavioural responses triggered by light encompass improved alertness and performance, as indexed by specific cortical responses to cognitive tasks in Photon Emission Tomography and functional Magnetic Resonance Imaging (fMRI) techniques [13]. However, dosage (intensity and duration), timing and wavelength of light for domestic use and in the workplace environments are difficult to define and may critically depend on environmental and the individual factors.

In a previous study, we have shown that exposure to gradually increasing light prior to awakening can counteract sleep restriction effects on well-being and cognitive performance across the day, leading to an optimized level of alertness, which impinges on enhanced performance on specific cognitive tasks tightly related to sustained levels of attention [14].

Most of the effects were visible on the first day after the sleep restriction night but not on the second day after two nights of sleep restriction, most likely due to the increase in sleep pressure.

The overall aim of the present study was to investigate whether dawn simulation light following sleep restriction, enhances performance according to cognitive domain and whether these effects are sustained during the entire day.

\section{Material and methods}

\subsection{Study participants}

Study volunteers were recruited through advertisements at different local universities and websites in Switzerland, Germany and France. Screening procedure began with a telephone interview, involving a detailed study explanation. All participants gave written informed consent before the start of the laboratory part. Study protocol, screening questionnaires and consent forms were approved by the local ethics committee (EKBB/Ethikkommission beider Basel, Switzerland) and conformed to the Declaration of Helsinki.

All applicants completed questionnaires about their sleep quality, life habits and health state. These questionnaires comprised a consent form, a general medical questionnaire, Beck Depression Inventory II [15], Epworth Sleepiness Scale [16], Horne Ostberg Morningness Eveningness Questionnaire [17], Munich Chronotype Questionnaire [18] and Pittsburgh Sleep Quality Index. Potential candidates with a Pittsburgh Sleep Quality Index score $>5$ were excluded from participation [19]. Further exclusion criteria were smoking, medication or drug consumption, body mass index $<19$ and $>28$, shift work and transmeridian flights within the last three months, as well as medical and sleep disorders. Since our study protocol included two nights of partial sleep restriction (restriction to 6-h), we also excluded participants with habitual sleep durations $<7-h$ and $>9-h$ [20], to minimize a possible confounding effects of sleep duration.

Eighteen young men (20-33 years old; mean + Standard Error of Mean: 23.1+.8) fulfilling all the criteria were enrolled in the study. A comprehensive toxicological analysis of urine for drug abuse was carried out before the study, along with an ophthalmologic examination to exclude volunteers with visual impairments.

One week before the study, participants were not allowed to drink excessive alcohol, and to consume caffeine or cacao containing drinks or meals (at most 5 alcoholic beverages per week, and 1 cup of coffee or 1 caffeine-containing beverage per day). They were also instructed to keep a regular sleep-wake schedule (bed and wake times within \pm 30 min of self-selected target time). Compliance to this outpatient segment of the study was verified by wrist actigraphy (actiwatch L, Cambridge Neurotechnologies, Cambridge, UK) and self-reported sleep logs.

The study was carried out during the winter season (January to March) in Basel, Switzerland, and comprised two segments, distributed in a balanced cross-over design, separated by at least 1 -week intervening period. The volunteers reported to the Centre for Chronobiology at the Psychiatric Hospital of the University of Basel on two occasions (control condition and one experimental conditions), where they stayed in individual windowless bedrooms with no information about time of day. Since we did not find significant effects on cognitive performance neither after the blue light exposure nor after the DsL exposure after the second night of sleep restriction, we decided to focus here on the first 24-h of the control condition and the DsL condition. The most likely explanation was that sleep pressure was too high after the second night of sleep restriction, and thus the morning light could not counteract its

A

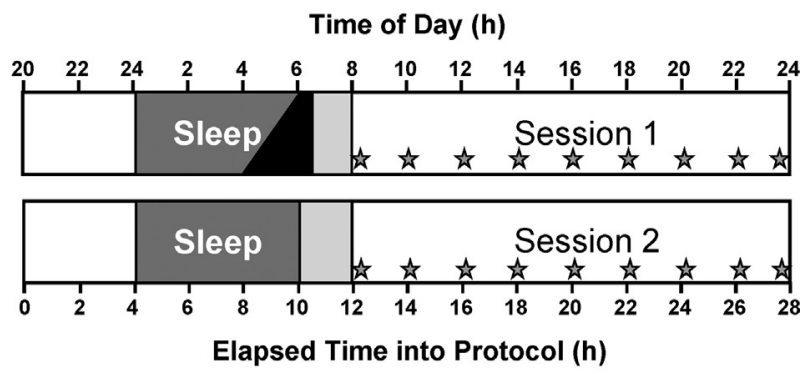

Continuous EEG, EOG, EMG, Heart Rate, measurement of body temperature and respiration

B

\section{Dawn Simulation Light spectrum}

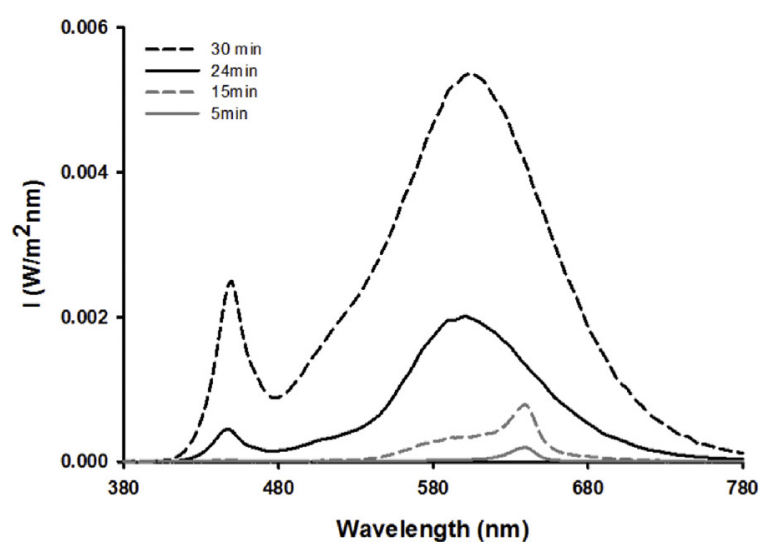

Fig. 1. (A) Protocol design. Two arms of a 6-h sleep restricted protocol with different morning light exposures. Elapsed time indication is relative to an arrival in the lab at 8 p.m. Time-of-day indication varied across all subjects but was given in the figure as an example (taken from the mean of the sleep/wake time from all participants). (B) Morning light device. Spectral composition (light wavelength by irradiance (W/m2nm)) of the Dawn Simulation Light at $5 \mathrm{~min}$ (grey solid), $15 \mathrm{~min}$ (grey dash), $24 \mathrm{~min}$ (black solid) and $30 \mathrm{~min}$ (black dash). 
detrimental effect. For more details, referred to Gabel et al. [14]. These 24-h include the first sleep restriction night (6-h) at 0 lx starting at the subject's habitual sleep time and followed by 18 -h of data recording during wakefulness. During the day, in both conditions, participants were exposed to dim light $(<8 \mathrm{~lx})$ during 2 -h after wake-up and to $40 \mathrm{~lx}$ for the remainder of the day until they went to bed (Fig. 1A). In both conditions, the investigator enters the room in the morning, 6-h after light off, to wake-up the participants.

The light treatment was administered after the sleep restriction night either with no additional light for the control condition or with a Dawn Simulation Light (DsL: LED prototype of Philips Wake-up Light HF3520, Philips Drachten, The Netherlands) (polychromatic light gradually increasing from 0 to $250 \mathrm{~lx}$ during $30 \mathrm{~min}$ before wake-up time; the light remains around $250 \mathrm{~lx}$ for $20 \mathrm{~min}$ after wake-up time), placed near the bed at eyes level (Fig. 1B). One participant could not be included in the analysis because of poor quality of the electroencephalogram recordings and noncompliance during cognitive testing. The remaining 17 volunteers underwent both light conditions in a balanced cross-over design as follows: DL-DsL for nine volunteers and DsL-DL for eight.

\subsection{Assessment of subjective ratings}

Subjective sleepiness was assessed every hour with a Visual Analogue Scale (100 mm scale). Likewise, after every test session participant had to indicate the effort, concentration and motivation they needed to perform the tests. A $100 \mathrm{~mm}$ effort visual analogue scale was used, ranging from "0:little" to "100:much" [21,22].

\subsection{Cognitive performance}

All tests were administered every 2 h during wakefulness, starting $30 \mathrm{~min}$ after wake up. The test battery comprised the Motor Tracking Task (MTT), Digit Symbol Substitution Test (DSST) [23], Paced Visual Serial Addition Task (PVSAT) [24,25], Sustained Attention to Response Task (SART) [26], Psychomotor Vigilance Task (PVT) [27], Simple Reaction Time Task (simRT) and Visual N-back Task (1,2,3-back) [28]. For detailed information about each test, please see Table 1 .

All the results are given relative to elapsed time after wake-up from restricted sleep taken at 6 a.m. Time-of-day indication was also added to be more informative and understandable for general public; it is an example calculated with the mean of the participants sleep/wake cycle, as they did not have the same scheduled time.

\subsection{Statistical analysis}

For all analyses, the statistical package SAS (version 9.1; SAS Institute, Cary, NC, USA) was used. Statistical analyses were carried out for each variable (subjective fatigue, motivation, concentration, effort, PVT, MTT, DSST, SimRT, SART, PVSAT and N-Back) separately with the mixed-model analysis of variance for repeated measures

Table 1

Description of the tasks in the cognitive test battery.

\begin{tabular}{|c|c|c|c|}
\hline Tests & Cognitive domain & Explanation of the tests & References \\
\hline Motor Tracking Task (MTT) & & $\begin{array}{l}\text { People need to track a computer-generated disc using the } \\
\text { mouse, along another computer-generated point for } 20 \mathrm{~s}\end{array}$ & \\
\hline $\begin{array}{l}\text { Digit Symbol Substitution } \\
\text { Test (DSST) }\end{array}$ & $\begin{array}{l}\text { Computer-based "cognitive } \\
\text { throughput" task (or attention-based } \\
\text { task) measuring clerical speed and } \\
\text { accuracy. The task becomes less } \\
\text { sensitive to changes in attention and } \\
\text { more sensitive to memory impairment. }\end{array}$ & $\begin{array}{l}\text { Participants are required to select a predefined digit in } \\
\text { response to each appearance of an abstract symbol (the key } \\
\text { linking symbols and digits are available on screen throughout } \\
\text { testing). They learn the coding relationships after some } \\
\text { experience. In this paced version of the task, } 8 \text { symbols are } \\
\text { presented for } 500 \text { ms, with an ISI of } 1500 \text { ms. Each symbol } \\
\text { occur an equal number of times. }\end{array}$ & Aster et al. [23] \\
\hline $\begin{array}{l}\text { Paced Visual Serial } \\
\text { Addition Task (PVSAT) }\end{array}$ & $\begin{array}{l}\text { Addition task heavily dependent on } \\
\text { frontal brain regions, involving } \\
\text { executive aspects of working memory }\end{array}$ & $\begin{array}{l}\text { Single digits ( } 1 \text { to } 9 \text { ) appear on screen and each must be added } \\
\text { to the digit which preceded it, and the resulting answer is } \\
\text { selected from an on-screen numerical keypad ("sum" of } \\
\text { adjacent pairs, not a total across all digits presented). Digits } \\
\text { were seen for } 1000 \mathrm{~ms} \text {, with inter-stimulus interval (ISI) of } \\
2000 \mathrm{~ms} \text { in between. }\end{array}$ & $\begin{array}{l}\text { Feinstein et al. [24]; } \\
\text { Nagels et al. [25] }\end{array}$ \\
\hline $\begin{array}{l}\text { Sustained Attention to } \\
\text { Response Task (SART) }\end{array}$ & $\begin{array}{l}\text { Sustained/divided attention task in } \\
\text { which inattention and inhibitory } \\
\text { processing can be measured } \\
\text { separately. Both types of performance } \\
\text { depend on the frontal cortex }\end{array}$ & $\begin{array}{l}\text { It's a computer-based task, used here as a Go/NoGo task, which } \\
\text { requires participants to monitor single digits presented rapidly } \\
\text { on screen, and respond to each one that appears (called Go } \\
\text { target), except for a particular pre-defined digit (called NoGo } \\
\text { target). Digits are presented for } 1000 \text { ms, with an inter-item } \\
\text { interval of } 2000 \text { ms, with } 15 \text { "targets" (where responses are } \\
\text { withheld) randomly interspersed with } 25 \text { distractors. }\end{array}$ & $\begin{array}{l}\text { Robertson et al. } \\
{[26]}\end{array}$ \\
\hline $\begin{array}{l}\text { Psychomotor vigilance task } \\
\text { (PVT) }\end{array}$ & $\begin{array}{l}\text { Sustained attention task, sensitive to } \\
\text { circadian variation and sleep loss It is } \\
\text { an approach of Dinges (Dinges, Pack } \\
\text { et al., 1997). }\end{array}$ & $\begin{array}{l}\text { The PVT involves a 5-min visual reaction time (RT) } \\
\text { performance test in which the subject is required to maintain } \\
\text { the fastest possible RT's to a simple auditory stimulus. Data } \\
\text { analyses focus on RT, errors, and signal detection theory } \\
\text { parameters. }\end{array}$ & Dinges et al. [27] \\
\hline $\begin{array}{l}\text { Simple Reaction Time Task } \\
\quad(\operatorname{simRT})\end{array}$ & $\begin{array}{l}\text { Indicator of speeded motor } \\
\text { performance. } \\
\text { Vary as a function of alertness and } \\
\text { sleep deprivation }\end{array}$ & $\begin{array}{l}\text { This task requires people to respond, as quickly as possible, to a } \\
\text { single, predictable stimulus. The participant rests his/her index } \\
\text { finger on a mouse, and having done so over the next } 1000 \mathrm{~ms} \text { a } \\
\text { word such as "Now!" appears, at which point the participant } \\
\text { moves the cursor to a designated target position. The time } \\
\text { taken from Now! To the onset to move the cursor from the } \\
\text { resting position reflects the participants' ability to detect that a } \\
\text { response is required, and, together with the subsequent time } \\
\text { taken to move the mouse to the target position, give us the RT. }\end{array}$ & \\
\hline $\begin{array}{l}\text { Visual N-back Task } \\
\text { (1,2,3-back) }\end{array}$ & Executive aspects of working memory & $\begin{array}{l}\text { Stimuli are presented individually on a computer screen, and } \\
\text { the participant must indicate (using the mouse), if the current } \\
\text { stimulus and the } n \text { stimuli prior to it match. The higher the n, } \\
\text { the stronger are the demands of executive functioning. Stimuli } \\
\text { are presented for } 500 \text { ms with an ISI of } 1500 \text { ms. The ratio } \\
\text { between match to non-match trials is of } 1: 2 \text { with } 30 \text { items. }\end{array}$ & Cohen et al. [28] \\
\hline
\end{tabular}


Table 2

Results of the analysis of the variance for different variables of subjective feeling for the time course of the study.

\begin{tabular}{|c|c|c|c|}
\hline \multirow[t]{2}{*}{ Variable } & \multicolumn{3}{|l|}{ Analysis of the variance } \\
\hline & Light & Time of day & Light $\times$ Time \\
\hline Effort & $F_{1,254}=0.14, p=.7059$ & $F_{8,254}=1.21, p=.2927$ & $F_{8,254}=51, p=.8485$ \\
\hline Concentration & $F_{1,254}=.69, p=.4061$ & $F_{8,254}=1.42, p=.1877$ & $F_{8,254}=1.08, p=.3806$ \\
\hline Motivation & $F_{1,254}=2.87, p=.0913$ & $F_{8,254}=1.56, p=.1377$ & $F_{8,254}=.45, p=.8927$ \\
\hline Fatigue & $F_{1,254}=00, p=.9996$ & $F_{8,254}=4.28, p<.0001$ & $F_{8,254}=1.07, p=.3830$ \\
\hline
\end{tabular}

(PROC MIXED), with within factors "light condition" (dim light [DL] versus dawn simulation light [DsL]) and "time-of-day" (all assessed time points).

A further analysis included the factor "group" was done, according to the subject's performance on the 3-back task. As this working memory paradigm presents a relatively high cognitive load it might be suitable to differ between high and low performers. Thus, we performed a median split and considered subjects presenting performances below the median of the overall group as low performers $(N=9)$ and those above as high performers $(N=8)$.

\section{Results}

\subsection{Assessment of subjective ratings}

No significant differences were found between light conditions for effort, concentration and motivation needed to perform the tasks. Similarly, subjective sleepiness induced by the test did not differ across the light conditions (Table 2). For detailed information on sleepiness, well-being and mood, please see [14].

\subsection{Cognitive performance}

\subsubsection{MTT}

Main effects of "light condition" and "time-of-day" were observed for the MTT (Table 3), such that after the DsL exposure participants were better at following the dot than after the DL exposure during the entire day (Fig 2B).

\subsubsection{DSST}

Main effects of "light condition" and "time-of-day" were also observed for DSST (Table 3) but in the opposite direction. Participants performed better after the DL exposure than after the DsL exposure throughout the day (Fig. 2A).

\subsubsection{PVSAT}

PVSAT performance did not yield significant differences neither for the main factors "light condition", nor for the interaction of "light condition $\times$ time of day" (Table 3 ). Only the factor "time of day" was significant such that participants improved their performance across the day independent of light treatment (data not shown in the figure).

\subsubsection{SART}

Main effect of "light condition" (Table 3) was observed for accuracy (correct answers for Go and NoGo targets), such that until 5-h of time awake, the performance was not significantly different irrespective of light settings, while 6-h after wake-time DsL improved performance along the day as compared to DL (Fig. 2D).

Besides, NoGo trials were analyzed as a proxy for inhibition control. It revealed a main effect of "light condition" ( $p=0.0013)$. Participants had less correct NoGo answers after the DsL exposure than after the DL exposure and mostly at the beginning of the day. Furthermore, the missed answers (missed Go answers) showed a main effect of "light condition" ( $p<0.0001)$, such that the DsL led to lower levels of missed answers than the DL. The reaction time for this task showed also a significant trend of the "light condition" effect $(p=0.0876)$, such that participants answered faster at the beginning of the day after the DsL.

\subsection{5. $P V T / \operatorname{sim} R T$}

The DsL exposure did not influence reaction time during the PVT (Table 3, data not shown in the figure) and performance was similar after both light exposures. However, the DsL decreased reaction time in the SimRT test throughout the day, as shown by a main effect of "light condition" and "time of day" (Table 3, Fig. 2C).

\subsection{6. $N-B A C K$}

The 1-Back showed a main effect of "light condition" (Table 3 ) for accuracy, driven by DsL exposure. After 4-h of elapsed time awake, DsL improved accuracy, with a stronger effect in the evening, while performance remained stable after DL exposure (Fig. 2E).

No significant differences were found between both light conditions in the 2-Back test (data not shown in the figure).

For the 3-Back test, we found a main effect of "light condition", such that participants had a higher rate of correct answers after a DsL than after a DL exposure, starting at the beginning of the day and remaining all the day (Fig. 2F).

Table 3

Results of the analysis of the variance for different variables of cognitive performance for the time course of the study.

\begin{tabular}{|c|c|c|c|}
\hline \multirow[t]{2}{*}{ Variable } & \multicolumn{3}{|l|}{ Analysis of variance } \\
\hline & Light & Time of day & Light $\times$ Time \\
\hline MTT & $F_{1,260}=1.76, p=.0079$ & $F_{8,260}=2.05, p=.0407$ & $F_{8,260}=.58, p=.7933$ \\
\hline DSST & $F_{1,254}=7.22, p=.0077$ & $F_{8,254}=2.44, p=.0148$ & $F_{8,254}=.25, p=.9800$ \\
\hline SimRT & $F_{1,271}=5.52, p=.0195$ & $F_{8,271}=3.07, p=.0025$ & $F_{8,271}=.33, p=.9554$ \\
\hline PVT & $F_{1,264}=3.31, p=.0700$ & $F_{8,264}=1.95, p=.0528$ & $F_{8,264}=.44, p=.8933$ \\
\hline SART & $F_{1,255}=5.07, p=.0252$ & $F_{8,255}=.28, p=.9731$ & $F_{8,255}=.31, p=.9601$ \\
\hline PVSAT & $F_{1,272}=0.96, p=.3277$ & $F_{8,272}=2.42, p=.0152$ & $F_{8,272}=.90, p=.5204$ \\
\hline 1-Back & $F_{1,255}=5.86, p=.0162$ & $F_{8,255}=.66, p=.7269$ & $F_{8,255}=.88, p=.5368$ \\
\hline 2-Back & $F_{1,255}=0.04, p=.8323$ & $F_{8,255}=.56, p=.8127$ & $F_{8,255}=1.12, p=.3497$ \\
\hline 3-Back & $F_{1,254}=16.69, p<.0001$ & $F_{8,254}=.48, p=.8716$ & $F_{8,254}=.16, p=.9952$ \\
\hline
\end{tabular}

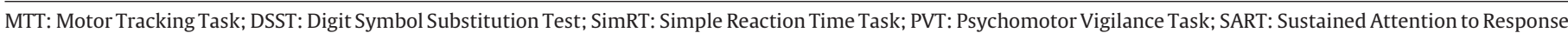
Task; PVSAT: Paced Visual Serial Addition Task; 1-2-3-Back: Visual N-back Task. 

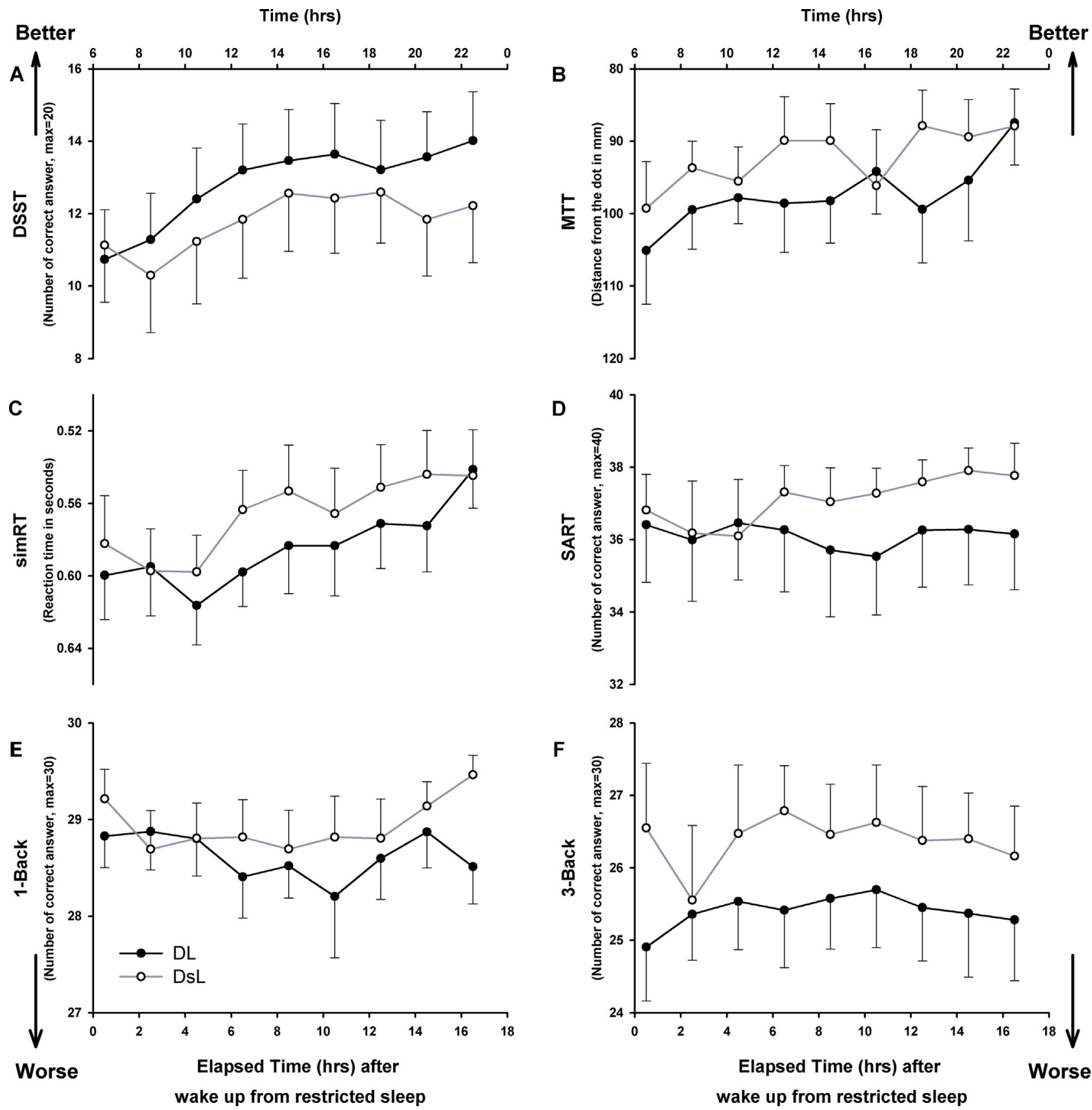

Fig. 2. Accuracy of the cognitive performance over the day.

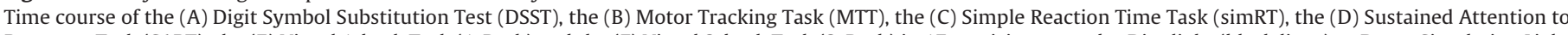

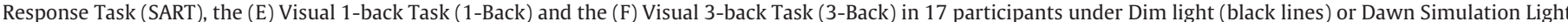

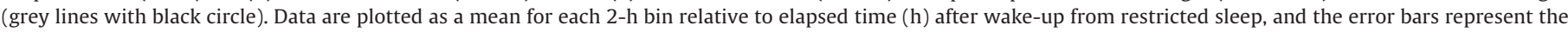
standard error of the mean. Elapsed time indication is relative to 6 a.m. wake-up time.

\subsubsection{Reaction times}

Furthermore, the composite score of the reaction time from those cognitive tests presenting higher cognitive load (n-Back, SART, PVSAT) was significantly lower after the DsL than after the DL (supplementary data).

\subsection{High vs. low performers}

Splitting the group according to high and low performance, a main effect of "light condition" was present in the low but not in the high performers, such that the DsL light improved performance in the PVSAT $(p=0.0096)$, the 3 -Back test $(p<0.0001)$ and the simRT test $(p=0.0064)$ compared to the DL condition (Fig. 3).

\section{Discussion}

Our data show that artificial morning light exposure, as indexed by DsL, has a task-dependent effect on cognitive performance under sleep restriction conditions, such that morning DsL significantly enhanced performance on attention-based tasks (SART, 1-verbal Back, and simRT). Furthermore, DsL significantly improved performance on the MTT that involves motor-based skills, and the 3 -verbal back, which mostly probes executive function. However, 
High performers
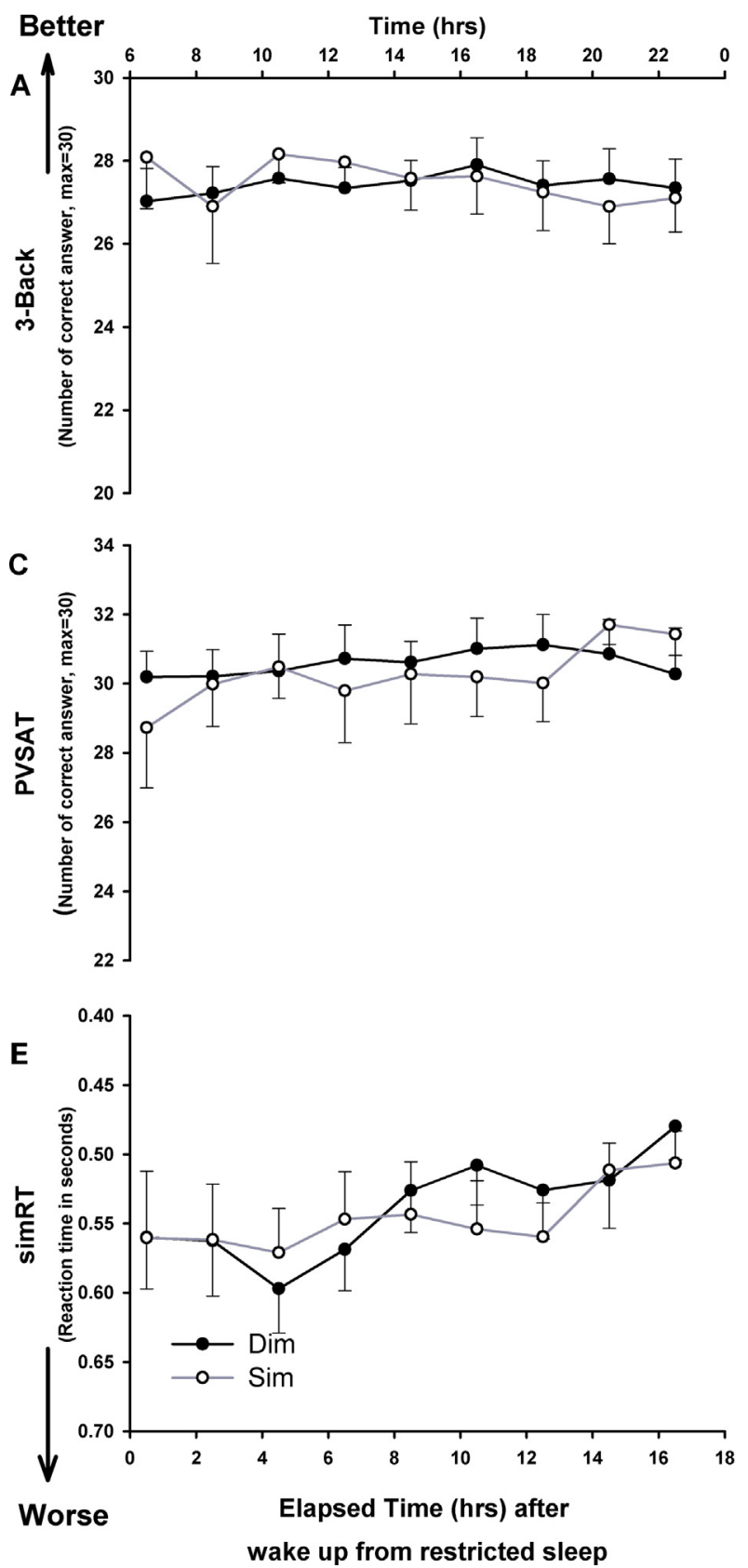
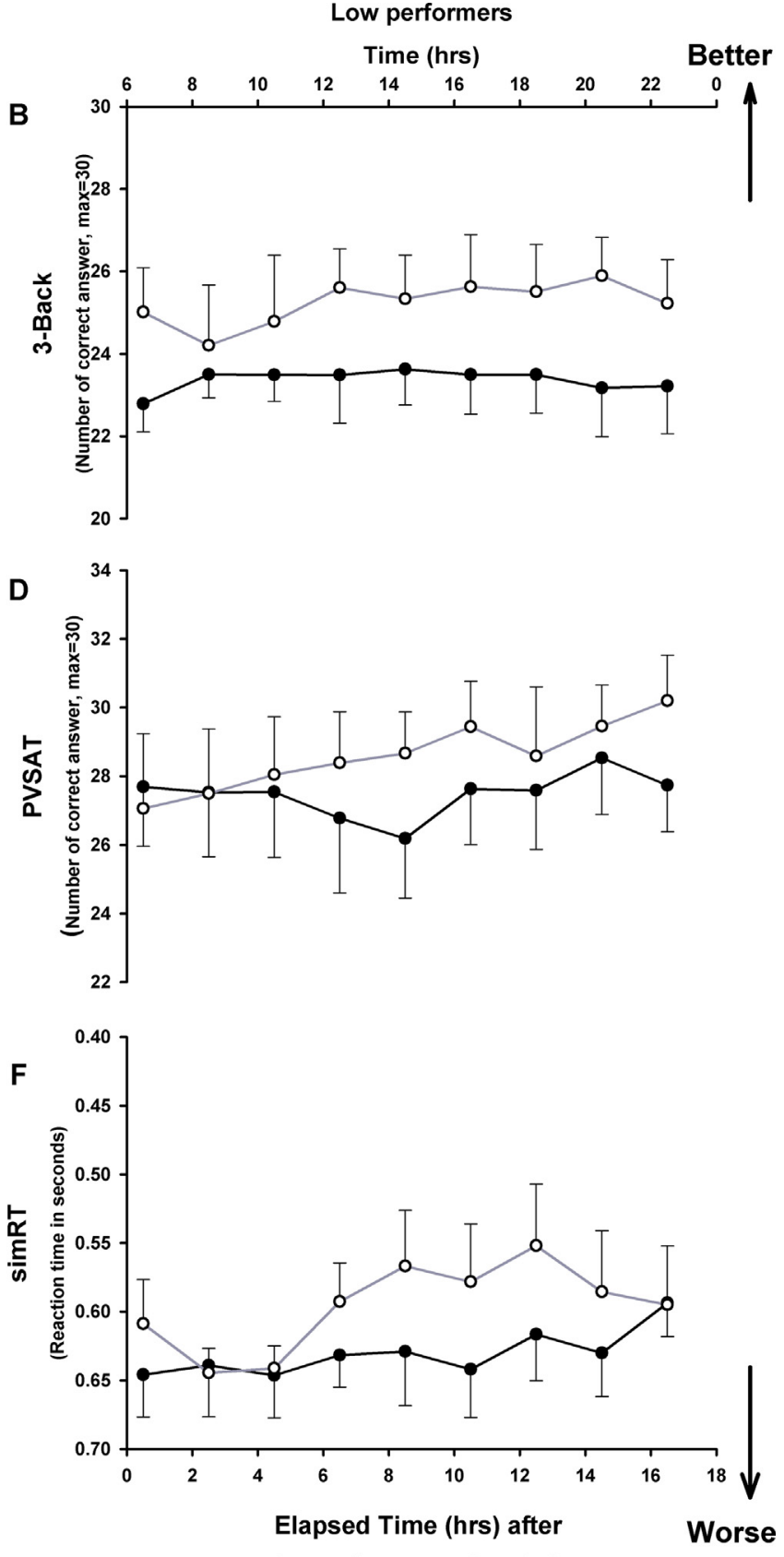

wake up from restricted sleep

Fig. 3. Accuracy of the cognitive performance over the day in the high and low performers.

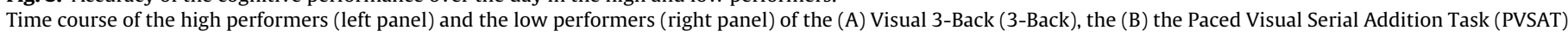

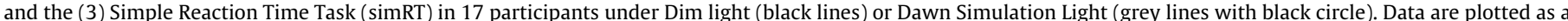

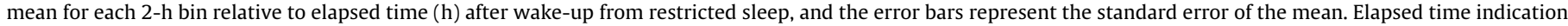
is relative to 6 a.m. wake-up time.

we also found better performance after the DL compared to the DsL for the DSST.

Our results corroborate our previous analysis that artificial morning dawn simulation light improves subjective perception of well-being and mood, as well as cognitive performance across the day under conditions of mild sleep restriction [14]. However, here we showed that the light effect on performance depends on the investigated cognitive domain, suggesting that different pathways may be implicated in this effect. Previous studies indicate that evening light exposure impacts on numerous domains of cognitive performance such as sustained attention [10], working memory and attention, as well as declarative memory [9].
Here we show that light exposure during the morning hours can significantly boost cognitive performance and maintain its stability throughout the day. Most studies on the effects of light in humans have been carried out during the night, when light is able to counteract the increasing sleep pressure, and thus significantly enhance or stabilize cognitive performance (for a review, see [29]). In this context, the modulatory mechanisms accounting for the light effects on cognition have been ascribed to its impact on sleep homeostasis and/or its indirect synchronizing/phase-shifting effects on the circadian timing system [10]. However, alternative mechanisms may bypass these systems, thus eliciting direct activating effects, particularly when light is timed during the day. 
Evidence for the latter arises from studies in which daytime performance increased after $30 \mathrm{~min}$ from light onset [30,31], from an fMRI study in which daytime light enhanced cognitive brain activity during an oddball task [32] and from our previous analysis in which morning light exposure (dawn simulation light) increased wellbeing and enhanced performances across the day [14]. Recently, another study showed the critical role of light for cognitive brain responses in emphasizing the evidence of a cognitive role for melanopsin, which may confer a form of "photic memory" to human cognitive brain function [33]. Collectively, these data suggest that light may modulate ongoing cortical activity involved in alertness, thus stimulating cognitive brain function.

One crucial brain region involved in the impact of light on cognition is the anterior hypothalamus, in locations compatible with the suprachiasmatic nucleus and the ventrolateral preoptic nucleus [34]. This region is postulated to be the primary link of the retina to the brain, thus mediating the effect of light on cognition [13]. Furthermore, numerous retinal projections extend to the lateral geniculate nucleus and also (albeit less) to the superior colliculus (SC). From the lateral geniculate nucleus, projections are sent to the primary visual cortex, which is the first processing site of the cortical visual pathway. Functionally, this pathway is classified in dorsal and ventral streams [35]. The former is associated to motion processing (medial temporal and superior temporal, and also parietal cortices), while the latter involves salient visual processing [36]. Interestingly, attention-related modulatory effects impact on both streams. Within the dorsal stream, attention is "encoded" by neurons involved in spatial attention/feature-based attention, such as the orientation of an object or a moving dot [36]. Concomitantly, motion-sensitive medial temporal and superior temporal areas are also involved in these processes, as well as higher cortical areas such as the intraparietal cortex [37]. We tentatively speculate that this particular network may be underlying the responses in the MTT task, and that light might play a modulatory role on this type of spatial attention/feature-based attention.

With respect to the ventral attention-based stream, a key structure involved in this attentional network is the SC, which receives direct input from the retina. This attention network is presumably shared with the intraparietal region [38], frontal eye fields [39], and visual cortices, through direct connections from the cortex to the SC, and indirectly from the SC to the cortex via the pulvinar [40]. The pulvinar (dorsal thalamic nuclei) is pivotal in attention modulation, presumably through the flow of information in the brain. It receives a direct retinal projection, and provides an indirect link between the suprachiasmatic nucleus and the prefrontal cortex [41]. Thus, it mediates arousal regulation, and an effect of light on the thalamus will probably result in a widespread cortical impact, such as on attention-based cognitive performance. Taken together, we postulate that these brain networks may underlie the modulatory light effects on numerous dimensions of attention tasks, such as on MTT, 1-Back, simRT and SART. It could also explain the lack of correct NoGo answers and the decrease of the missed Go answers in the SART test after the DsL exposure. Effectively, arousal levels are more prominent at the beginning of the day and might even be more pronounced after a light exposure, which explains response accuracy at the beginning of the day. However, different tasks require different levels of arousal for optimal performance [42]. For example, difficult or intellectually demanding tasks may require a lower level of arousal (to facilitate concentration), whereas tasks demanding stamina or persistence may be performed better with higher levels of arousal (to increase motivation) [43]. According to this concept, it could be argued that the arousal level after the DsL exposure was too high to inhibit the participant's answers in the SART task, which is in accordance with the increase of the reaction time in this task during the first hours after wake time. This leads to a speed-accuracy trade-off in the inhibitory process, meaning that light exposure induces higher excitability resulting in faster reaction time and thus in lack of inhibition control.

Our PVT data did not parallel earlier findings of a beneficial light effect $[10,30]$. One possibility is that, contrary to our design, these studies challenged sustained attention performance either during light exposure and did not specifically explore carry-over effects or after a prolonged daytime light exposure. However they are comparable to the findings from Van de Werken [44], showing that the reaction times under DsL were similar to the one under control condition. One could argue that the PVT needs a higher and, more probably, a sustained level of arousal than the SART test - which is not reached here - to get to optimal performance levels, since this task is much more monotonous with much fewer stimuli (e.g., [45]). The absence of a significant effect could be traced back to the fact that we compared reaction times after one night of sleep restriction between two light conditions. This is different to other studies, comparing rested wakefulness to sleep deprived or sleep restricted conditions. Similarly, the DSST showed the contrary of the expected effect, such that participants were slower in reacting and less accurate. We have no straightforward explanation for the opposing effect of the DSST. It cannot be due to a simple learning effect across conditions as we counterbalanced the order and we did not detect a significant interaction between time of day and condition. Neither the observed pattern can be attributed to general impairments in working memory functions across the day under DsL as we found better performance under DsL for the n-Back test. These two tasks were the first two tasks administered within each testing sessions, thus, a certain time-on-task effect can be ruled out, and a floor effect of light impact because of high demand is equally implausible.

Interestingly, our data also show a significant DsL effect on the 3-verbal back task. This task challenges working memory, which refers to the individual capacity to temporarily maintain active relevant information to perform an ongoing task [46,47]. This task involves the need to continuously update and inhibit information, and also has an attentional component to it. Thus, one possible explanation to the DsL effects is that light may enhance performance on the 3-back task via its impact on the basic attention component in this complex working-memory task. However, at the behavioural level, it is not possible to dissect out the components existing within this task. Thus, light might also impact on the executive component of this task. The 3-back task probes executive control, and its cerebral correlates involve the bilateral posterior parietal cortex, premotor cortex, dorsal cingulate/medial premotor cortex, dorsolateral prefrontal cortex and ventrolateral prefrontal cortex [48]. Light may have a modulatory effect on these anatomical structures, thus impacting on executive brain responses [49].

Furthermore, light has greater beneficial effects on the low performers (stratified according to the 3-Back results) compared to the high performers in the 3-back, PVSAT and simRT. However, it is important to consider a potential ceiling effect for the high performers; as the sleep restriction was not that severe, these participants could easily cope with this and thus, keep their high level of performance. Thereby, the impact of light might have been difficult to detect.

Interestingly, the low performers on the 3-back who profit most from the light intervention are also the ones who experience a detrimental effect of light on their reaction times in the PVT. This is somewhat contra-intuitive, as we previously showed that light leads to faster reaction time [10]. However, it could indicate that the low performers are more cautious in their task performance under the effect of light, and therefore profit in a complex task, where accuracy is important, but decline in a more basic task where fast reactions are required. Clearly, it indicates that the light effect is dependent on the cognitive domain and obviously also acts differentially depending on how well someone performs. 


\section{Conclusion}

Our results collectively indicate that short exposure to gradually increasing morning light (DsL) just before the end of the partially restricted night episode, may significantly enhance performance, particularly in cognitive tasks associated to attention. In a broader context, these findings point to strategies that may directly optimize attention-related cognition in real-life settings, particularly when individuals are sleep restricted.

\section{Supplementary data}

Reaction time from the cognitive tests over the day. Time course of the composite of reaction time of the Paced Visual Serial Addition Task (PVSAT), Sustained Attention to Response Task (SART) and Visual 1-2-3-back Task (1-2-3-Back) in 17 participants under Dim light (black lines) or Dawn Simulation Light (grey lines with black circle). Data are plotted as a mean for each 2 -h bin relative to elapsed time $(\mathrm{h})$ after wake-up from restricted sleep, and the error bars represent the standard error of the mean. Elapsed time indication is relative to 6 a.m. wake-up time.

\section{Conflicts of interest}

The authors report no conflicts of interest. The authors alone are responsible for the content and writing of the paper.

\section{Acknowledgments}

We thank Dr. Götz for medical screenings, Claudia Renz, Marie-France Dattler and Giovanni Balestrieri for their help in data acquisition, Amandine Valomon for her help in recruiting volunteers and, of course, the volunteers to participating. This research was supported by Philips Consumer Lifestyle, Drachten, The Netherlands.

\section{Appendix A. Supplementary data}

Supplementary data associated with this article can be found, in the online version, at http://dx.doi.org/10.1016/j.bbr.2014.12.043.

\section{References}

[1] Dijk DJ, Duffy JF, Czeisler CA. Circadian and sleep/wake dependent aspects of subjective alertness and cognitive performance. I Sleep Res 1992:1:112-7.

[2] Silva EJ, Wang W, Ronda JM, Wyatt JK, Duffy JF. Circadian and wake-dependent influences on subjective sleepiness, cognitive throughput, and reaction time performance in older and young adults. Sleep 2010;33:481-90.

[3] Bonnet MH. Performance and sleepiness as a function of frequency and placement of sleep disruption. Psychophysiology 1986;23:263-71.

[4] Van Dongen HP, Maislin G, Mullington JM, Dinges DF. The cumulative cost of additional wakefulness: dose-response effects on neurobehavioral functions and sleep physiology from chronic sleep restriction and total sleep deprivation. Sleep 2003;26:117-26.

[5] Belenky G, Wesensten NJ, Thorne DR, Thomas ML, Sing HC, Redmond DP, et al. Patterns of performance degradation and restoration during sleep restriction and subsequent recovery: a sleep dose-response study. J Sleep Res 2003; $12: 1-12$.

[6] Durmer JS, Dinges DF. Neurocognitive consequences of sleep deprivation. Semin Neurol 2005;25:117-29.

[7] Jones K, Harrison Y. Frontal lobe function, sleep loss and fragmented sleep. Sleep Med Rev 2001;5:463-75.

[8] Walker MP. Sleep-dependent memory processing. Harvard Rev Psychiatry 2008; 16:287-98.

[9] Cajochen C, Frey S, Anders D, Spati J, Bues M, Pross A, et al. Evening exposure to a light-emitting diodes (LED)-backlit computer screen affects circadian physiology and cognitive performance. J Appl Physiol 2011;110:1432-8.

[10] Chellappa SL, Steiner R, Blattner P, Oelhafen P, Gotz T, Cajochen C. Non-visual effects of light on melatonin, alertness and cognitive performance: can blueenriched light keep us alert? PLoS One 2011;6:e16429.

[11] Berson DM, Dunn FA, Takao M. Phototransduction by retinal ganglion cells that set the circadian clock. Science 2002;295:1070-3.
[12] Smith MR, Revell VL, Eastman CI. Phase advancing the human circadian clock with blue-enriched polychromatic light. Sleep Med 2009;10:287-94.

[13] Vandewalle G, Maquet P, Dijk DJ. Light as a modulator of cognitive brain function. Trends Cogn Sci 2009;13:429-38.

[14] Gabel V, Maire M, Reichert CF, Chellappa SL, Schmidt C, Hommes V, et al. Effects of artificial dawn and morning blue light on daytime cognitive performance, well-being, cortisol and melatonin levels. Chronobiol Int 2013;30:988-97.

[15] Beck AT, Ward CH, Mendelson M, Mock J, Erbaugh J. An inventory for measuring depression. Arch Gen Psychiatry 1961;4:561-71.

[16] Johns MW. A new method for measuring daytime sleepiness: the Epworth sleepiness scale. Sleep 1991:14:540-5.

[17] Horne JA, Ostberg O. A self-assessment questionnaire to determine morningness-eveningness in human circadian rhythms. Int J Chronobiol 1976;4:97-110.

[18] Roenneberg T, Wirz-Justice A, Merrow M. Life between clocks: daily temporal patterns of human chronotypes. J Biol Rhythms 2003;18:80-90.

[19] Buysse DJ, Reynolds 3rd CF, Monk TH, Berman SR, Kupfer DJ. The Pittsburgh Sleep Quality Index: a new instrument for psychiatric practice and research. Psychiatry Res 1989;28:193-213.

[20] Aeschbach D, Cajochen C, Landolt H, Borbely AA. Homeostatic sleep regulation in habitual short sleepers and long sleepers. Am J Physiol 1996:270:R41-53.

[21] Verwey W, Veltman H. Detecting short periods of elevated workload: comparison of nine assessment techniques. J Appl Psychol Applied 1996;3: 270-85.

[22] Dunham KJ, Shadi S, Sofko CA, Denney RL, Calloway J. Comparison of the repeatable battery for the assessment of neuropsychological status effort scale and effort index in a dementia sample. Arch Clin Neuropsychol 2014;29: 633-41.

[23] Aster Mv NA, Horn R WAIS-III. Wechsler Adult Intelligence Scale Frankfurt/M., Germany: Harcourt 2006.

[24] Feinstein A, Brown R, Ron M. Effects of practice of serial tests of attention in healthy subjects. J Clin Exp Neuropsychol 1994;16:436-47.

[25] Nagels G, Geentjens L, Kos D, Vleugels L, D’Hooghe MB, Van Asch P, et al. Paced visual serial addition test in multiple sclerosis. Clin Neurol Neurosurg 2005; 107:218-22.

[26] Robertson IH, Manly T, Andrade J, Baddeley BT, Yiend J. 'Oops!': performance correlates of everyday attentional failures in traumatic brain injured and normal subjects. Neuropsychologia 1997;35:747-58.

[27] Dinges DF, Pack F, Williams K, Gillen KA, Powell JW, Ott GE, et al. Cumulative sleepiness, mood disturbance, and psychomotor vigilance performance decrements during a week of sleep restricted to $4-5$ hours per night. Sleep 1997;20:267-77.

[28] Cohen JD, Perlstein WM, Braver TS, Nystrom LE, Noll DC, Jonides J, et al. Temporal dynamics of brain activation during a working memory task. Nature 1997;386:604-8.

[29] Chellappa SL, Gordijn MC, Cajochen C. Can light make us bright? Effects of light on cognition and sleep. Prog Brain Res 2011;190:119-33.

[30] Phipps-Nelson J, Redman JR, Dijk DJ, Rajaratnam SM. Daytime exposure to bright light, as compared to dim light, decreases sleepiness and improves psychomotor vigilance performance. Sleep 2003;26:695-700.

[31] Ruger M, Gordijn MC, Beersma DG, de Vries B, Daan S. Time-of-day-dependent effects of bright light exposure on human psychophysiology: comparison of daytime and nighttime exposure. Am J Physiol Regul Integr Comp Physiol 2006;290:R1413-20

[32] Vandewalle G, Balteau E, Phillips C, Degueldre C, Moreau V, Sterpenich V, et al. Daytime light exposure dynamically enhances brain responses. Curr Biol 2006:16:1616-21.

[33] Chellappa SL, Ly JQ Meyer C, Balteau E, Degueldre C, Luxen A, et al. Photic memory for executive brain responses. Proc Natl Acad Sci U S A 2014;111: 6087-91.

[34] Perrin F, Peigneux P, Fuchs S, Verhaeghe S, Laureys S, Middleton B, et al. Nonvisual responses to light exposure in the human brain during the circadian night. Curr Biol 2004;14:1842-6.

[35] Goodale MA, Milner AD. Separate visual pathways for perception and action. Trends Neurosci 1992;15:20-5.

[36] Baluch F, Itti L. Mechanisms of top-down attention. Trends Neurosc 2011:34:210-24

[37] Martinez-Trujillo JC, Treue S. Feature-based attention increases the selectivity of population responses in primate visual cortex. Curr Biol 2004;14: 744-51.

[38] Bisley JW, Goldberg ME. Attention, intention, and priority in the parietal lobe. Annu Rev Neurosci 2010;33:1-21.

[39] Thompson KG, Bichot NP. A visual salience map in the primate frontal eye field. Prog Brain Res 2005;147:251-62.

[40] Shipp S. The functional logic of cortico-pulvinar connections. Philos Trans Roy Soc London Ser B: Biol Sci 2003;358:1605-24.

[41] Sylvester CM, Krout KE, Loewy AD. Suprachiasmatic nucleus projection to the medial prefrontal cortex: a viral transneuronal tracing study. Neuroscience 2002; 114:1071-80.

[42] Yerkes R, Dodson J. The relation of strength of stimulus to rapidity of habitformation. J Comparat Neurol Psychol 1908;18:459-82.

[43] Broadhurst PL. Emotionality and the Yerkes-Dodson law. J Exp Psychol 1957;54:345-52.

[44] Van De Werken M, Gimenez MC, De Vries B, Beersma DG, Van Someren EJ Gordijn MC. Effects of artificial dawn on sleep inertia, skin temperature, and the awakening cortisol response. J Sleep Res 2010;19:425-35. 
[45] Lim J, Dinges DF. Sleep deprivation and vigilant attention. Ann N Y Acad Sci 2008;1129:305-22.

[46] Baddeley A. The concept of working memory: a view of its current state and probable future development. Cognition 1981:10:17-23.

[47] Schmidt C, Collette F, Cajochen C, Peigneux P. A time to think: circadian rhythms in human cognition. Cogn Neuropsychol 2007;24:755-89.
[48] Sala-Llonch R, Arenaza-Urquijo EM, Valls-Pedret C, Vidal-Pineiro D, Bargallo N, Junque C, et al. Dynamic functional reorganizations and relationship with working memory performance in healthy aging. Front Hum Neurosci 2012;6:152.

[49] Vandewalle G, Gais S, Schabus M, Balteau E, Carrier J, Darsaud A, et al. Wavelength-dependent modulation of brain responses to a working memory task by daytime light exposure. Cereb Cortex 2007;17:2788-95. 